\title{
RF Design of The Temperature Compensation For The Quiescent Working Point of The Amplifier
}

\author{
Zhiqi Piao, Yuemin Ning, Qi Wang \\ China Electronics Technology Instruments Co.Ltd \\ Science and Technology on Electronic Test \& Measurement Laboratory \\ *765467910@qq.com
}

Keywords: power amplifier; bias circuit; temperature compensation

Abstract. The linearity and gain of RF power amplifier are greatly affected by the quiescent working point of the amplifier. In practice, the threshold opening voltage of the power amplifier changes with the temperature rise, which leads to the change of the quiescent working current, and which makes the linearity and gain deteriorate. In this paper, two kinds of temperature compensation circuits applied to the quiescent working point of power amplifier are presented. The temperature compensation of power amplifier is realized by using temperature sensor and operational amplifier, ensure that the power amplifier can maintain good linearity and gain at different temperature, and the circuit structure is simple and easy to debug.

\section{Introduction}

Power amplifier is widely used in radar and communications, and the linearity of broadband high power amplifier is becoming a research hotspot. In practical application, it is necessary to consider the influence of temperature change on the parameters of power amplifier. Temperature changes will directly affect the quiescent current work, and changes the type of work power amplifier, which changes of power amplifier gain, efficiency and linearity, even directly burned equipment for high power amplifier. Therefore, the design of the temperature compensation circuit is the key to the stability of the power amplifier. This paper proposes two temperature compensation circuits for power amplifiers. The circuit has the advantages of simple structure, high compensation precision and easy to debug, which can be easily put into practical application.

\section{Amplifier thermal behavior}

The quiescent current management of amplifier has a strong effect on its performance because the critical RF performance parameters, such as intermodulation distortion products, are dependent on the quiescent current level [1].

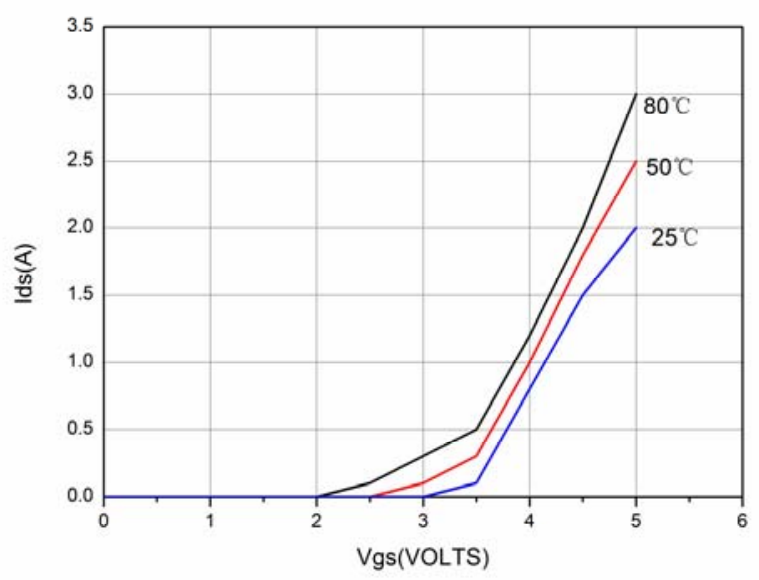

Figure 1. IDS versus VGS and Case Temperature 
Figure 1 presents the quiescent current thermal behavior of a 30 Watt LDMOS device. The drain-source current is strongly impacted by temperature. In Class AB and for a given VGS, the IDQ rises with temperature. Consequently, the shapes of the AM/AM response as well as linearity are affected by temperature [2].

The control goal of the bias circuitry is to maintain the quiescent current constant in all amplifier stages even if the environmental and device temperature changes significantly. Carefully selecting the control strategy will optimize the devices linearity performance and enable the built-in quiescent current thermal tracking circuit to work properly.

\section{Temperature compensating circuit}

3.1 Circuit design (1). The temperature compensation circuit of the power amplifier is realized through the combination of a temperature sensor and operational amplifier.

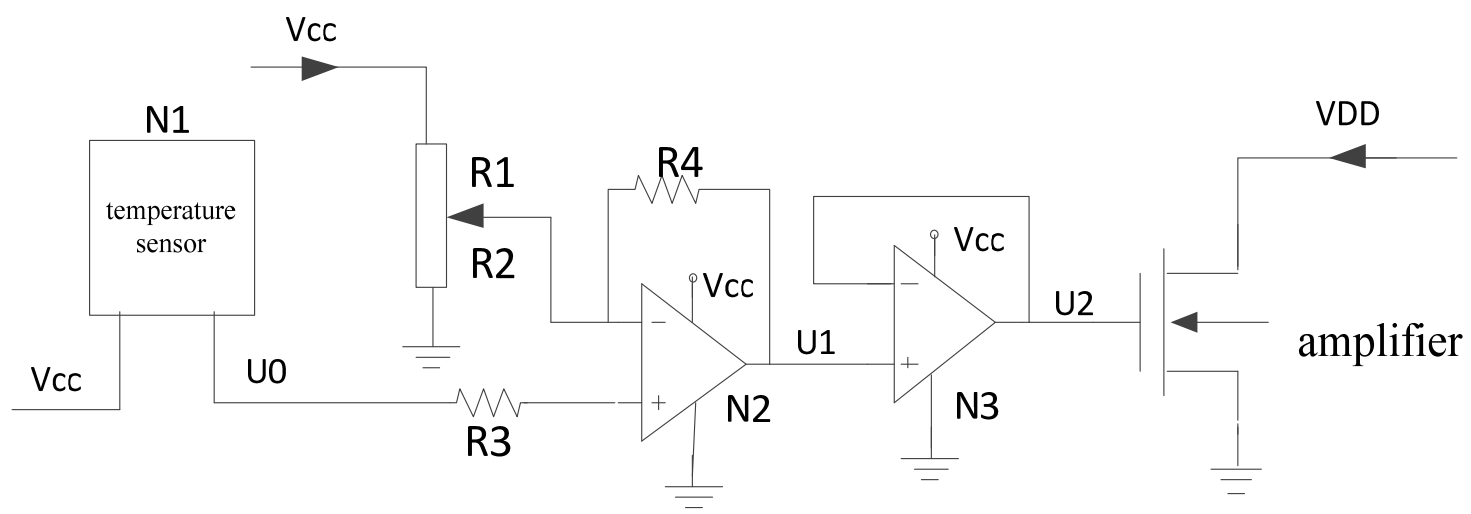

Figure 2. Thermal tracking schematic with its bias circuit

The circuit is shown in Figure 2. First of all, the temperature sensor N1 is attached to the printed board of the power amplifier, and the output voltage U0 increases linearly with temperature. The output voltage U0 passes the operational amplifier N2, Output U1 as following equation.

$$
\frac{V_{c c}-U_{0}}{R_{1}}=\frac{U_{0}}{R_{2}}+\frac{U_{0}-U_{1}}{R_{4}}
$$

U1 passes the follower N3, and the output U2 is connected to the Gate of the power amplifier. By formula (1), the voltage values required for the power amplifier Gate can be obtained through the resistance selection of R1, R2 and R4. When the temperature increases, the output U1 decreases linearly with the increase of temperature. Because $\mathrm{U} 1=\mathrm{U} 2$, the gate voltage of the amplifier is also reduced. In this way, the temperature rise can be offset, resulting in the increase of the working current of the power amplifier, which stabilizes the static working point.

3.2 Circuit design (2). The bias controller uses a small-signal PNP transisior (mounted in contact with the baseplate) to monitor temperature and generate $\mathrm{a}+8 \mathrm{mV} /{ }^{\circ} \mathrm{C}$ compensating voltage [3]. The circuit is shown in Figure 3.

The gate current is relatively large due to the supply requirements of the built-in thermal tracking circuit. The thermal tracking circuit contains a thermal tracking transistor with its gate and drain connected together and its source connected to ground, along with several voltage settings and current limiting resistors.

As a result of the additional components in this thermal tracking circuit, the gate current draw is in the milliamp range (not in the micro amp range) and follows the change of the drain current. The thermal tracking circuit is physically located on the die right next to the active RF amplifier die area so its operating temperature is closely tied to that of the main amplifier circuit. 


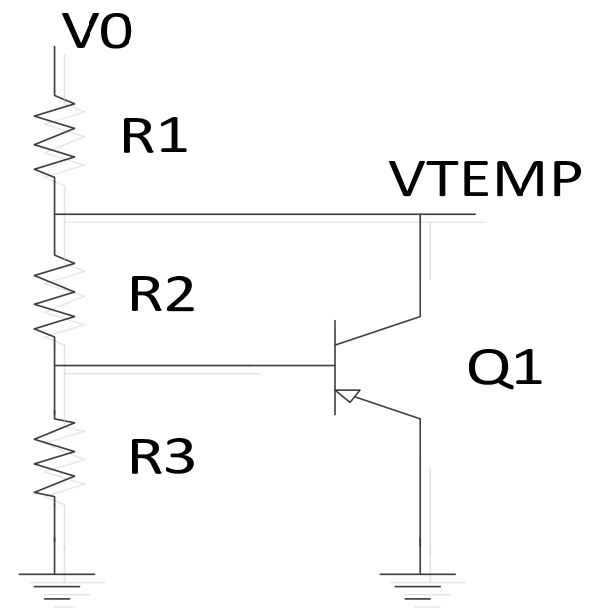

Figure 3. Temperature compensating circuit

A variable voltage derived from the V0 supply is sunned with the temperature monitor voltage to generate a temperature-compensated gate voltage. R6 and R7 are selected to set the desired gate voltage trim range, and R4 is selected to provide the desired amount of temperature compensation (see Figure 4).

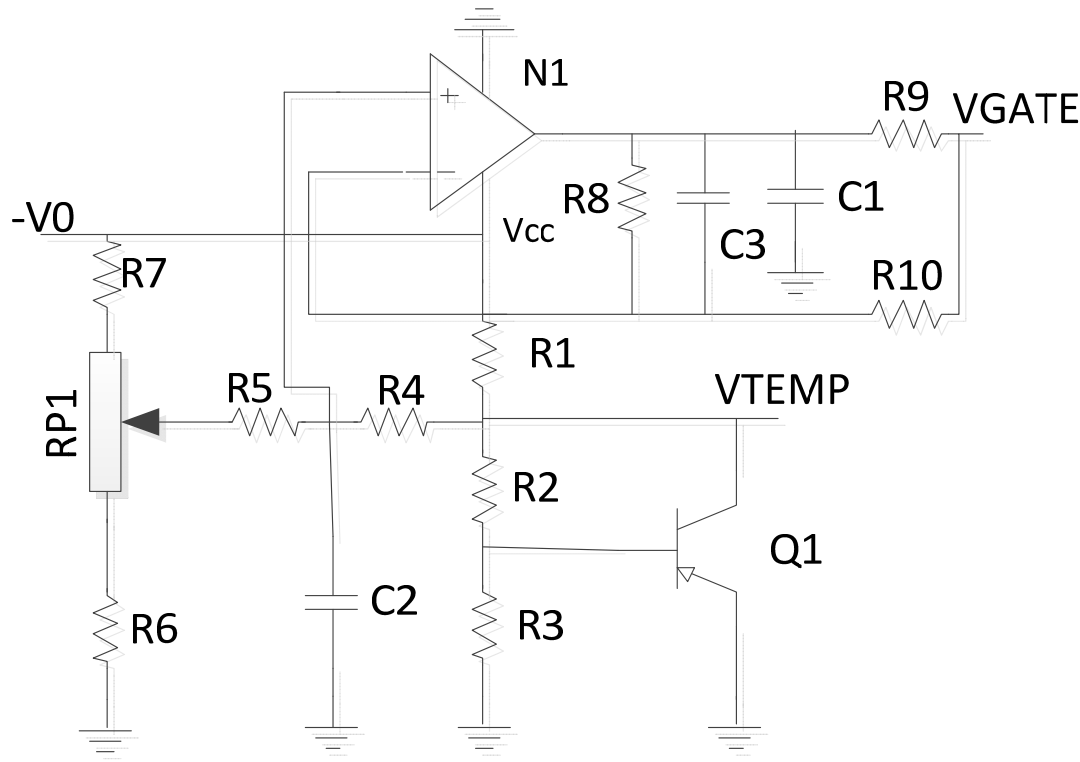

Figure 4. Gate voltage adjustment circuit

$\mathrm{N} 1$ is a dual rail-to-rail high-current operational amplifier chosen because it is stable into any capacitive load. It can deliver more than $100 \mathrm{~mA}$ of output current to meet the positive gate current requirements of most amplifiers [4].

The output impedance of the bias source is low (less than $1 \Omega$ ) because of the feedback around operational amplifier N1. However, HEMT applications usually require a series gate resistor of $5 \Omega$ to $20 \Omega$, instead of a bias inductor, to ensure low frequency device stability.

Because the amplifier quiescent gate current can increase at high temperatures, a significant voltage drop can be developed across this gate resistor, increasing Vgs by $200 \mathrm{mV}$ or more. This can be a serious problem because the increased gate voltage can push the amplifier operating point into a region of instability or even cause thermal runaway.

One solution is to provide DC feedback from the amplifier gate terminal to the operational amplifier inverting input, allowing it to compensate for voltage dropped across gate resistor R9. Feedback through R10 compensates for voltage dropped across R9. To minimize RF non-linearity and memory effects, make sure that $1 /(\mathrm{R} 10 * \mathrm{C} 8)$ is less than the lowest modulation frequency of the RF signal [5]. 


\section{Conclusions}

The temperature compensation circuit introduced in this paper has been successfully applied in practical circuit design and has stable performance. However, due to the dispersion of parameters, each power amplifier will be different. Before the large-scale application, the design of the temperature compensation circuit to verify, has achieved the best results.

\section{Acknowledgment}

The work is fully supported by the Funds of Science and Technology on Electronic Test \& Measurement Laboratory (Grant 6142001010101). Authors wish to thank the Science and Technology on Electronic Test \& Measurement Laboratory for all financial and technical support for this paper.

\section{References}

[1] Olivier Lermbeye, Tean Christophe Nanan. Effect of Temperature on High-Power RF LDMOS Transistors. Applied Microwave \& Wireless, August 2002.

[2] Modeling Thermal Effects in RF LDMOS Transistors. MOTO Applicatons Notes. AN1941.

[3] Curtice W, PI6J,Bridges D, et al. A new dynamic electro-thermal nonlinear model for silicon RF LDMOS FET [C]. IEEE MTT-S international microwave symposium, IEEE MTT-S International TUIA-2. Anaheim CA, 1999.

[4] Xu Weixin: Reliability design of electric product: Electric industry press. Beijing. 2000.

[5]F.H.Raab, “Class-F power amplifier swith maximally flat wavforms,”IEEE Trans.Microw.Theory Tech. vol. 45, no. 11, pp. 2007-2012, Nov. 1997. 